\title{
Kant, le droit cosmopolitique et la société civile des nations
}

Marc Belissa et Florence Gauthier

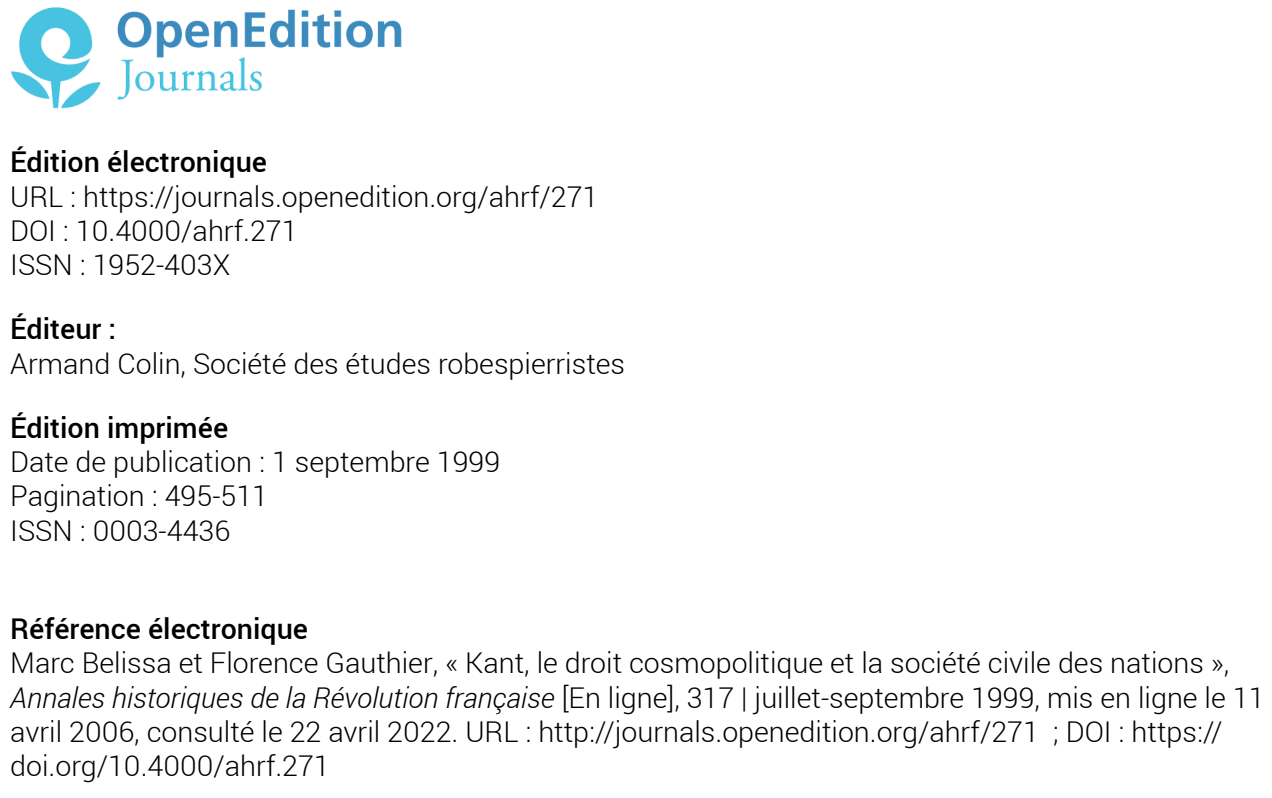

Ce document a été généré automatiquement le 22 avril 2022.

Tous droits réservés 


\title{
Kant, le droit cosmopolitique et la société civile des nations
}

\author{
Marc Belissa et Florence Gauthier
}

\begin{abstract}
«On peut dire que cette institution universelle et perpétuelle de la paix n'est pas une simple partie, mais constitue la fin ultime tout entière de la doctrine du droit dans les limites de la simple
\end{abstract} raison. ")

1 De 1784, date des Idées d'une Histoire universelle du point de vue cosmopolitique, jusqu'en 1795, date de publication du Zum Ewigen Frieden (Projet de paix perpétuelle), Kant s'est attaché à définir un droit cosmopolitique, c'est-à-dire un droit s'appliquant aux nations dans leurs relations respectives mais aussi aux individus en tant que membres de la "grande famille " de l'humanité. La réflexion de Kant s'insère dans le grand débat philosophique sur les relations entre les peuples, débat qui a ses origines au $\mathrm{XvI}^{\mathrm{e}}$ siècle et qui connaît une phase nouvelle au xvine siècle, en particulier depuis la paix d'Utrecht en $1713^{1}$. Les questions posées par ce débat sont les suivantes : l'humanité est-elle naturellement vouée à la paix ou la nature de l'homme provoque-t-elle inéluctablement des affrontements entre les nations? Comment concevoir un droit entre les peuples qui puisse pacifier leurs rapports ou du moins atténuer les effets de la nature belligène de l'homme? Et enfin, est-il possible de définir pratiquement la perspective d'une "société civile des nations ", c'est-à-dire d'une société dans laquelle ce nouveau droit des nations serait un lien éthique et jouerait le même rôle que le droit civil et politique dans les sociétés particulières?

L'insociable sociabilité et la perspective cosmopolitique

Dès 1784 Kant considère les transformations subies par le genre humain comme des étapes du progrès de l'humanité vers une forme cosmopolitique « réalisant les idéaux de la raison ». La nature possède un but propre qu'elle réalise dans les actions des hommes, qui sont à la fois libres et déterminés par le sens de l'histoire, elle utilise l'antagonisme des hommes au sein de la société pour mener à bien le développement de ses dispositions. Kant désigne cet antagonisme par la formule de l'insociable sociabilité 
qui réalise la synthèse dialectique de l'opposition traditionnelle entre la "sociabilité naturelle" des jusnaturalistes et la "guerre de tous contre tous" de Hobbes. L'insociable sociabilité, précise Kant, est « l'inclination à entrer en société, inclination qui est cependant doublée d'une répulsion générale à le faire, menaçant constamment de désagréger cette société ${ }^{2}$ ». Cette opposition dialectique est porteuse de progrès car cette "tension " est le moteur des actions humaines. Le dessein de la nature ne peut être réalisé que dans la société. C'est pourquoi «le problème essentiel pour l'espèce humaine, celui que la nature contraint l'homme à résoudre, c'est la réalisation d'une société civile administrant le droit de façon universelle ${ }^{3}$ ». " Une société, ajoute Kant, dans laquelle la liberté soumise à des lois extérieures se trouvera liée au plus haut degré possible à une puissance irrésistible, c'est-à-dire à une organisation civile d'une équité parfaite, doit être pour l'espèce humaine la tâche suprême de la nature ${ }^{4}$ ». Or " le problème de l'établissement d'une constitution civile parfaite est lié au problème de l'établissement de relations régulières entre les États, et ne peut pas être résolu indépendamment de ce dernier ». La société civile des nations ne peut donc être construite sans un changement radical dans la constitution de chacune des sociétés particulières. Kant rejoint ici Rousseau qui, dans le Jugement de la paix perpétuelle de l'abbé de Saint-Pierre, avait critiqué la manière «naïve » de l'abbé d'envisager le futur de l'Europe. Il faut "républicaniser» les constitutions civiles des États pour pouvoir construire la société des nations (Fœedus Amphyctionum précise Kant). Si l'idée de paix perpétuelle a été raillée à la suite du projet de l'abbé de Saint-Pierre, c'est parce qu'elle a été envisagée comme une construction immédiate, et non comme un processus. Pour Kant, la paix perpétuelle est une perspective et non un état statique.

3 L'insociable sociabilité des hommes se retrouve à l'échelle des nations, mais, ruse suprême de la nature, même les guerres poussent les hommes vers la paix ! En effet, pour les besoins du développement de leur puissance, les États entretiennent des relations permanentes (diplomatie, commerce etc.), ce qui prouve selon Kant, l'existence du «dessein secret» de la nature. La paix perpétuelle, conséquence d'une constitution cosmopolitique accomplie, n'est donc pas une "rêverie de visionnaire", mais le mouvement objectif de l'humanité: «Un jour enfin, en partie par l'établissement le plus adéquat de la constitution civile sur le plan intérieur, en partie sur le plan extérieur par une convention et une législation communes, un état de choses s'établira qui, telle une communauté civile universelle, pourra se maintenir par lui-même ${ }^{5}$.»

La définition d'un nouveau droit des gens

4 Pour construire cette société civile des nations, il est indispensable de concevoir un droit nouveau. En effet, en sortant de l'état de nature et créant la société civile, les hommes acceptent de se soumettre à un droit commun, mais les États sont toujours entre eux dans l'état de nature, c'est-à-dire dans un état non-juridique où la précarité règne ${ }^{6}$. Depuis le XVI ${ }^{e}$ siècle, les philosophes et les " publicistes » tentent de définir les éléments d'un droit des gens - c'est-à-dire d'un droit s'appliquant aux relations entre les sociétés humaines. En effet, il existe bien un ensemble de traités, de conventions et de coutumes tacitement ou explicitement admises qui règlent les rapports entre les États. Ce corpus du droit des gens s'est construit par le jeu de l'accroissement des relations entre les États, c'est pourquoi il concerne essentiellement la conduite dans la guerre, la forme des traités de paix, les relations commerciales ou les immunités diplomatiques. Ce droit des gens existant entre les États est-il identique au droit naturel, en dérive-t-il, 
ou bien n'est-il qu'un droit conventionnel et coutumier sans légitimité morale transcendante?

$5 \quad \mathrm{Au}$ XVII ${ }^{\mathrm{e}}$ siècle, Grotius insiste sur l'aspect positif du droit des gens sans pour autant le détacher du droit naturel. Hobbes et Pufendorf identifient totalement le droit naturel et le droit des gens, car les États sont totalement dans l'état de nature du fait de l'inexistence d'un arbitre pour régler les différends entre les nations. Locke propose une solution qui exerce une grande influence sur les hommes des Lumières. Pour l'auteur des Traités du gouvernement civil, le droit des gens est à la fois un droit positif et une perspective éthique à réaliser dans les rapports de peuple à peuple ${ }^{7}$. Le droit des gens est donc une forme particulière du droit naturel, celui qui s'applique entre les sociétés civiles. Montesquieu ou Mably reprennent ce concept et rejettent l'idée grotienne d'un droit des gens essentiellement positif. Face à l'énigme du droit des gens, Rousseau est plus radical, l'ensemble des règles admises entre les États ne saurait avoir de légitimité transcendante. Il n'existe pas de véritable droit des gens : "Quant à ce qu'on appelle communément le droit des gens, il est certain que, faute de sanction, ses lois ne sont que des chimères plus faibles encore que la loi de nature, celle-ci parle au moins au cœur des particuliers au lieu que, le droit des gens, n'ayant d'autre garant que l'utilité de celui qui s'y soumet, ses décisions ne sont respectées qu'autant que l'intérêt les confirme ${ }^{8}$. $"$

6 L'attitude de Kant est contradictoire, à l'image de celle de la plupart des philosophes des Lumières. En effet, il considère d'un côté que les traités entre les souverains ou que les règles tacites adoptées entre les nations européennes en matière de droit de la guerre ou de droit de conquête ne sont pas fondés sur les vrais principes et ne sont donc que des bribes de coutume ne portant pas le caractère d'un véritable droit, mais dans le même temps, il estime que ces règles humanitaires sont l'expression du «dessein secret » de la nature affleurant dans les textes diplomatiques à l'insu de leurs rédacteurs.

7 La double nature du droit des gens - droit positif entre les États et droit naturel des individus en tant que membres de l'humanité - pose donc un problème philosophique de fond. Bentham tente de le résoudre en 1789 en créant un nouveau concept : le droit international, conçu comme une jurisprudence entre les nations. Bentham n'ignore pas la nouveauté introduite dans ce débat :

" The word international, it must be acknowledged, is a new one; though it is hoped, sufficiently analogous and intelligible. It is calculated to express, in a more significant way, the branch of law which goes commonly under the name of law of nations : an appelation so uncharacteristic, that, were it not the force of custom, it would seem rather to refer to internal jurisprudence ${ }^{9}$. "

Mais ce nouveau concept ne rend pas complètement le sens originel du terme «droit des gens ». La référence au droit naturel disparaît au profit d'une approche positive. En créant ce nouveau terme, Bentham néglige le concept de citoyenneté universelle. Si le droit «international» est la forme exclusive des relations entre les peuples, l'appartenance à la "grande famille » du genre humain disparaît en tant que catégorie juridique. Les États sont les médiateurs exclusifs du droit entre les peuples.

9 Face à ce même problème, Kant choisit une solution diamétralement opposée à celle de Bentham : le droit des gens existant doit être complété par la proclamation d'un droit cosmopolitique attaché à tous les individus. C'est cette solution qui est proposée dans les textes de 1793 et 1795 . 
La société civile des nations et la Paix perpétuelle

En 1793, Kant revient sur cette question dans: Sur l'adage: cela est peut-être juste en théorie mais ne vaut pas pour la pratique ${ }^{10}$. Le chapitre III, qui s'intitule « de la relation de la théorie à la pratique dans le droit des gens dans une perspective universelle philanthropique c'est-à-dire cosmopolitique », interroge: "Faut-il aimer le genre humain dans son ensemble? Ou bien est-ce un objet que l'on doit considérer avec irritation, auquel on souhaite sans doute (pour se garder de devenir misanthrope) toute espèce de bien, mais sans jamais en attendre de lui et dont on doit plutôt détourner les yeux ${ }^{11} »$ ? En d'autres termes, la perspective d'une fraternité universelle pratique estelle politique, ou bien doit-elle rester le "rêve d'un homme de bien "? Cette question rejoint selon nous celle de Robespierre dans le débat constitutionnel de 1793: les hommes en société sont-ils isolés comme des "troupeaux de créatures humaines parqués sur un coin du globe » ou bien sont-ils une fraction de "l'immense famille »? Existe-t-il des « devoirs de fraternité qui unissent tous les hommes et toutes les nations ${ }^{12}$ »? La réponse réside pour Kant dans l'existence de "dispositions qui autorisent à conclure que l'espèce progressera toujours vers le mieux, et que le mal des temps présents et passés se perdra dans le bien des temps futurs ». Ces dispositions prouvent que la progression vers le mieux peut être interrompue, mais jamais rompue. De même que l'état de guerre initial a poussé les hommes à constituer des sociétés civiles, les guerres continuelles entre ces sociétés doivent les conduire "même contre leur volonté, dans une constitution cosmopolitique » qui ait la forme «d'un état juridique de fédération selon un droit des gens dont il a été convenu en commun ${ }^{13}$ ".

11 Mais des obstacles se dressent sur cette voie. Le principal est la forme du «pouvoir fédératif » dominant en Europe ${ }^{14}$. Il faut donc que chaque État soit «intérieurement organisé de telle façon que ce ne soit pas le chef de l'État, à qui au fond la guerre ne coûte rien (car il la conduit aux frais d'un autre, à savoir le peuple) mais le peuple, à qui elle coûte personnellement, qui ait la voix décisive pour dire s'il doit oui ou non y avoir guerre ${ }^{15}$ ». Cette idée - déjà présente chez Mably ou d'Holbach - était un topos de la philosophie des Lumières dont les Constituants se sont largement inspirés dans le débat sur le droit des gens ${ }^{16}$. Kant prend donc ici clairement position en faveur du contrôle du droit de guerre et de paix par le peuple (faisant écho à la discussion de mai 1790 à l'Assemblée constituante), c'est-à-dire pour une constitution républicaine au sens propre du terme dans laquelle la guerre et la paix, étant une chose commune relèvent de la décision du pouvoir législatif.

12 «L'équilibre des puissances »- présenté par les apologistes de la diplomatie d'Ancien Régime comme un mécanisme pacificateur - ne peut pas être la solution au problème de la constitution cosmopolitique, seul un nouveau droit des gens peut apaiser les rapports entre les sociétés :

« La nature humaine n'apparaît nulle part moins aimable que dans le rapport que les peuples pris comme totalité entretiennent entre eux. [...] La volonté de s'assujettir ou de se diminuer réciproquement dans ses biens est toujours présente; et les préparatifs de défense, qui rendent la paix souvent encore plus oppressante et plus dévastatrice pour la prospérité intérieure que la guerre elle-même, ne peuvent jamais se relâcher. Or, il n'y a face à cela pas d'autre solution qu'un droit des gens fondé sur des lois publiques que la force vient soutenir et auxquelles chaque État devrait se soumettre. [...] Car une paix générale, qui durerait en vertu de ce qu'on appelle la balance des forces en Europe est une pure chimère, comme la maison de Swift qui avait été construite par un architecte en si parfait accord avec toutes les lois de l'équilibre qu'elle s'effondra aussitôt qu'un moineau vint s'y poser ${ }^{17}$. » 
13 Ainsi, face au problème de la constitution conjointe de la polis et de la cosmopolis (F. Gauthier) ${ }^{18}$, Kant répond - comme une partie des révolutionnaires français - par la perspective juridique du droit des gens et par la constitution de sociétés civiles républicaines.

14 Le Projet de paix perpétuelle couronne cette réflexion et constitue une véritable synthèse du débat philosophique des Lumières sur les relations entre les peuples à la lumière de l'expérience révolutionnaire immédiate ${ }^{19}$. Le Zum Ewigen Frieden fait d'ailleurs figure de modèle pour la génération des disciples de Kant ${ }^{20}$; et dans les années qui suivent sa parution, une intense production philosophique répond au maître de Königsberg ${ }^{21}$. La rédaction du projet est contemporaine des négociations franco-prussiennes du traité de Bâle, mais, comme on l'a vu, une partie de ses éléments est déjà présente dans des textes antérieurs.

Dans son projet de 1795, Kant prend soin de se démarquer des accusations « d'utopisme ». La paix perpétuelle est considérée comme une perspective pratique. Le texte se compose de six « articles préliminaires » contenant les conditions politiques de la réalisation de la paix perpétuelle, de «trois articles définitifs " et de deux «suppléments ». La forme adoptée par Kant est une parodie des textes diplomatiques de son temps. L'inévitable article secret ne fait pas défaut, mais son contenu est volontairement ironique : il définit justement la transparence nécessaire aux relations entre les peuples !

Les six articles préliminaires constituent une critique en règle de la "politique » des États européens :

«1. Nul traité de paix ne peut être considéré comme tel, si l'on s'y réserve secrètement quelque sujet de recommencer la guerre. 2. Aucun État (petit ou grand, cela ne fait rien ici) ne peut être acquis par un autre, par voie d'héritage, d'échange, d'achat ou de donation. 3 . Les armées permanentes doivent entièrement disparaître avec le temps. 4. On ne doit pas contracter de dettes nationales en vue des intérêts extérieurs de l'État. 5. Aucun État ne doit s'immiscer de force dans la constitution et le gouvernement d'un autre État. 6. Nul État ne doit se permettre, dans une guerre avec un autre, des hostilités qui rendraient impossible, au retour à la paix, la confiance réciproque, comme par exemple, l'emploi d'assassins, d'empoisonneurs, la violation d'une capitulation, l'excitation à la trahison dans l'État auquel il fait la guerre, etc. ${ }^{22} »$

Cette énumération est parsemée d'indices et de références parfaitement intelligibles pour les lecteurs contemporains de Kant ${ }^{23}$. Le premier article condamne les pratiques «machiavéliques» de la diplomatie d'Ancien Régime, le second la " politique privée » des rois et la guerre de conquête, le troisième fait clairement référence au danger de l'armée permanente, le quatrième est une condamnation du système politico-financier de l'Angleterre, le cinquième reprend l'article 119 de la Constitution de 1793, et enfin, le sixième renvoie au droit de la guerre, mais constitue aussi une critique à peine voilée de la conduite de la guerre par la Première coalition ( $c f$. l'excitation à la trahison à Toulon...).

18 Ces six articles constituent pour Kant autant d'impératifs catégoriques, réalisables immédiatement ou à moyen terme. Il commente chacun d'entre eux de manière ironique. La diplomatie, qui n'a pour but que « l'accroissement continu de sa puissance, par quelque moyen que ce soit », doit disparaître ainsi que les Pactes de familles, qui permettent aux États de "s'épouser entre eux ». L'armée permanente est condamnée mais Kant admet les exercices militaires pour garantir les citoyens des agressions 
extérieures, claire référence à la Révolution française. Enfin les États ont le droit de se liguer contre une puissance qui fait la guerre à crédit, allusion transparente à l'Angleterre.

19 Kant précise ce qu'il entend par la guerre de conquête dans le second article préliminaire. Un État n'est ni un avoir ni une chose mercantile, mais une société d'hommes ayant une personnalité juridique du fait du contrat social primitif. Ce contrat primitif délégitime tout passage au pouvoir d'un autre État, quelle qu'en soit la forme :

«Un État n'est pas un patrimoine, comme le sol où il se trouve. C'est une société d'hommes, dont l'État seul ose disposer en maître. C'est un tronc qui a ses propres racines. L'incorporer à un autre État, comme une simple greffe, c'est le réduire, de personne morale qu'il était, à l'état d'une chose; ce qui contredit l'idée du contrat social, sans lequel on ne saurait concevoir de droit sur un peuple ${ }^{24}$.»

Kant distingue l'État comme personne morale qui a des racines propres et inaliénables, du droit de gouverner qui peut être modifié, de façon illégitime par la force, ou même de façon consentie, sans pour autant perdre ses racines. Cette distinction délégitime la prétention d'un gouvernement à la souveraineté, qui demeure un bien commun enraciné dans le terreau vivant d'une société d'hommes. La souveraineté-racine d'une société ouvre ici sur la légitimation de la résistance à l'oppression dans tous les cas où le droit de gouverner cherche à s'approprier la souveraineté.

Dans un texte postérieur, Kant précise le cas de conquête-colonisation comme usurpation de la souveraineté-racine du peuple irlandais. Après avoir énoncé l'interdit de la guerre offensive, il en précise trois formes:

«Aucune guerre entre États indépendants ne peut être une guerre punitive (bellum punitivum). En effet, il ne peut y avoir punition que dans la relation entre un supérieur (imperantis) et un subordonné (subditum), laquelle relation n'est pas celle des États entre eux. Et la guerre ne saurait non plus être une guerre d'extermination (bellum internecinum) ni une guerre d'asservissement (bellum subjugatorium) qui serait l'anéantissement moral d'un État [dont alors le peuple se fonderait dans la masse du peuple vainqueur, ou tomberait en servitude] ${ }^{25}$."

Développant la question du droit qui fait suite à la guerre, Kant condamne explicitement la conquête de type colonial en prenant l'exemple passé d'Athènes et celui actuel de l'Angleterre en Irlande :

«L'État vaincu ou ses sujets ne perdent point leur liberté civile du fait de la conquête de leur pays, au sens où celui-ci serait ravalé au rang de colonie et eux au rang de serfs, car autrement il y aurait eu guerre punitive, laquelle est en ellemême contradictoire. Une colonie ou province est un peuple qui a certes sa propre constitution, sa législation, son sol, où les ressortissants d'un autre État ne sont que des étrangers, mais sur lequel pourtant cet autre État détient le pouvoir exécutif. Ce dernier s'appelle la métropole. L'État sous tutelle est dominé par la métropole, mais pourtant il se gouverne lui-même (civitas hybrida) - à travers son propre parlement, au besoin sous la présidence d'un vice-roi. Tel fut le statut d'Athènes par rapport à différentes îles et tel est aujourd'hui celui de la Grande-Bretagne par rapport à l'Irlande. A fortiori est-il impossible de dériver le servage et sa légitimité de la domination d'un peuple par la guerre, car il faudrait admettre pour cela une guerre punitive. Et l'on peut moins encore admettre un servage héréditaire, lequel est absurde, surtout parce que la culpabilité attachée au crime de quelqu'un ne saurait être héritée ${ }^{26}$. " 
Domenico Losurdo a attiré l'attention sur la critique kantienne de la conquête coloniale comme forme du « plus grand despotisme concevable». Rappelons son analyse du texte que nous venons de citer :

«Le sens de cette prise de position est clair : malgré l'obtention en 1782, à la suite d'une autre vague révolutionnaire, de l'autonomie législative, malgré d'autres concessions en 1793, l'Irlande continuait d'être une colonie: les termes qu'utilise Kant, «État-fille » (Tochterstaat) et «État-mère » (Mutterstaat) soulignent la dureté de la condamnation de l'Angleterre à propos de la question irlandaise. Kant ne déclare-t-il pas que le «gouvernement paternel » qui traite ses sujets en « enfants mineurs » constitue «le plus grand despotisme concevable »? En ce cas, c'est toute une nation, et une nation européenne, qui est privée de ses droits. Un acte absolument dénué de justification. Même à la suite d'une défaite militaire, « l'État vaincu ou ses sujets ne perdent point leur liberté civile du fait de la conquête de leur pays, au sens où celui-ci serait ravalé au rang de colonie et eux au rang de serfs ». Pourtant, l'Irlande, vaincue et soumise par l'Angleterre, est tombée au rang de colonie. Si la Révolution française était justifiée... à titre de simple réforme par en haut, la révolution irlandaise l'était, elle, comme guerre de libération nationale 27. "

critique de cette forme de conquête coloniale donne lieu à un développement concernant les colonies esclavagistes européennes. De même que la guerre d'asservissement est illégitime, un contrat de soumission ne peut engager quiconque à la perte de ses droits. Si l'homme est une fin en soi, il ne peut servir d'instrument à un autre : «Nul ne peut s'engager par un contrat à une dépendance telle qu'il cesse par là d'être une personne; car ce n'est qu'en qualité de personne qu'on peut passer un contrat. »

Kant compare un contrat dont les clauses de services sont indéterminées à l'esclavage des Africains dans les plantations d'Amérique :

«Or, il semble bien qu'un homme puisse s'engager envers un autre à fournir certains services de nature licite mais indéterminés quant au degré, aux termes d'un contrat de louage, et devenir ainsi simple sujet (subjectus) sans être serf (servus); mais ce n'est là qu'une fausse apparence. En effet, si le maître est habilité à user à son gré des forces de son sujet, il peut arriver (comme cela se passe pour les Nègres dans les îles à sucre) qu'il les épuise jusqu'à la mort ou jusqu'au désespoir, et dans ce cas le sujet s'est véritablement livré à son maitre comme sa propriété, ce qui est impossible. »

De même il est également impossible, c'est-à-dire contraire au droit, de naître dans l'asservissement sous prétexte d'hérédité :

«Et l'on ne peut pas davantage revendiquer l'enfant d'un serf à cause des frais que son éducation a occasionnés, étant donné que l'éducation est un devoir absolu des parents, et dans le cas où ceux-ci ont été serfs, celui des maîtres, qui, tout en prenant possession de leurs sujets, ont aussi pris en charge leurs devoirs ${ }^{28}$. »

27 Chacun des trois "articles définitifs " correspond à l'un des "niveaux " juridiques nécessaires à la construction de la paix perpétuelle : droit civil, droit des gens et droit cosmopolitique. Leur réalisation est une perspective politique : Kant assigne aux hommes le devoir éthique de réaliser la paix conformément à la raison pure. L'état de paix n'est pas la cessation des hostilités, c'est un état juridique qui doit être construit. Pour cela, «la constitution civile de chaque État doit être républicaine» (premier article définitif), et "il faut que le droit des gens soit fondé sur une fédération d'États libres " (deuxième article définitif). Kant rejette expressément l'idée d'un super-État, tel que celui d'Anarchasis Cloots. La perspective de la paix perpétuelle doit se construire sur 
une fédération de peuples républicains. Le droit des gens tel qu'il existe, ne peut pas jouer le rôle que lui assigne Kant dans la construction de la paix perpétuelle, car le droit des gens, " entendu dans le sens de droit de guerre ", n'offre proprement aucun sens. Il n'est pas un droit, mais une collection de «maximes particulières ». Pour Kant, il ne saurait y avoir de droit de la guerre, car celle-ci est contraire à la loi morale. Comme la plupart des philosophes du XvIII ${ }^{\mathrm{e}}$ siècle, il considère d'ailleurs Grotius et Pufendorf comme de "tristes consolateurs ». Toutefois, « cet hommage que chaque État rend à l'idée du droit (du moins en paroles) ne laisse pas de prouver qu'il y a dans l'homme une disposition morale, plus forte encore, quoiqu'elle sommeille pour un temps, à se rendre maître un jour du mauvais principe qui est en lui (ce qu'il ne peut nier). Autrement, les États qui veulent se faire la guerre ne prononceraient jamais le mot droit, à moins que ce fût par ironie ${ }^{29}$. "

Le droit cosmopolitique complète les deux premiers niveaux : « Le droit cosmopolitique doit se borner aux conditions d'une hospitalité universelle" (troisième article définitif). Ce droit « considère les hommes et les États, dans leurs relations extérieures et dans leur influence réciproque comme citoyens d'un État universel de l'humanité ${ }^{30}$ ", il concerne les hommes en tant que citoyens du monde. Kant insiste sur le fait qu'il ne s'agit pas de philanthropie, mais du droit que possède chaque homme de ne pas être traité en ennemi dans un pays qui n'est pas le sien ${ }^{31}$. Le droit cosmopolitique n'est donc pas de nature «philosophique » comme le lui reprochera G. F. de Martens dans un texte polémique, mais bien de nature juridique ${ }^{32}$. Les violations du droit d'humanité concernent tous les hommes de quelque pays qu'ils soient :

«Les relations (plus ou moins étroites) qui se sont établies entre tous les peuples de la terre, ayant été portées au point qu'une violation du droit commise en un lieu se fait sentir dans tous, l'idée d'un droit cosmopolitique ne peut plus passer pour une exagération fantastique du droit; elle apparaît comme le complément nécessaire de ce code non écrit qui, comprenant le droit civil et le droit des gens, doit s'élever jusqu'au droit public des hommes en général, et par là jusqu'à la paix perpétuelle, dont on peut se flatter, mais à cette seule condition, de se rapprocher continuellement ${ }^{33}$.»

Le droit d'hospitalité donne lieu à une critique fondamentale de la politique des puissances européennes. Le droit d'hospitalité est très précisément limité à un simple droit de visite justifié par deux principes; le premier est « le droit qu'a l'étranger, à son arrivée dans le territoire d'autrui, de ne pas y être traité en ennemi», tant qu'il n'offense personne. Le second est «le droit qu'a tout homme de se proposer comme membre de la société, en vertu du droit de commune possession de la surface de la terre sur laquelle, en tant que sphérique, ils ne peuvent se disperser à l'infini; il faut donc qu'ils se supportent les uns à côté des autres, personne n'ayant originairement le droit de se trouver à un endroit de la terre plutôt qu'à un autre ${ }^{34}$ ».

C'est alors l'appartenance au genre humain qui légitime la demande de visite et celle de devenir membre d'une société (dans certaines conditions toutefois) comme partie intégrante du droit des gens concernant ici les personnes et non les États.

31 Ce droit de tout homme à la commune possession de la surface de la terre ne saurait être confondu avec une appropriation du sol et reste à construire à des conditions que l'actualité indique à Kant. Tout d'abord, le droit de visite n'est pas un droit d'accueil mais une simple demande de visite qu'une personne fait à une société et peut alors être refusée. Les causes de refus envisagées par Kant ici sont de deux ordres. Des comportements inhospitaliers comme ceux que l'on peut rencontrer sur les côtes 
barbaresques « où l'on s'empare des navires des mers avoisinantes, et où l'on réduit en esclavage les marins échoués ", ou encore chez les Bédouins «l'on considère comme un droit de piller ceux qui approchent des tribus nomades ». Toutefois, en dépit de ces comportements, le droit d'hospitalité peut chercher à se construire à condition qu'il se limite à «fixer les conditions sous lesquelles on peut essayer de former des liaisons avec les indigènes d'un pays. De cette manière des régions éloignées les unes des autres peuvent contracter des relations amicales, sanctionnées enfin par des lois publiques, et le genre humain se rapprocher insensiblement d'une constitution cosmopolitique 35 ".

De ces comportements inhospitaliers à caractère défensif, Kant distingue l'agressivité spécifique des "nations commerçantes de l'Europe" qui violent le droit de visite, pensent et agissent en conquérants au mépris des pays et des peuples et commettent des crimes qui font d'elles un danger menaçant la paix dans le monde. Kant précise que le mot découvrir signifie, pour ces nations commerçantes de l'Europe en Amérique, en Afrique, en Asie, conquérir :

« À quelle distance de cette perfection ne sont pas les nations civilisées et surtout les nations commerçantes de l'Europe ! À quel excès d'injustice ne les voit-on pas se porter, quand elles vont découvrir des pays et des peuples étrangers! (ce qui signifie chez elles les conquérir). L'Amérique, les pays habités par les Nègres, les îles à épices, le Cap, etc., furent pour eux des pays sans propriétaires, parce qu'ils comptaient les habitants pour rien. Sous prétexte de n'établir dans l'Hindoustan que des comptoirs de commerce, ils y débarquèrent des troupes étrangères, et par leur moyen ils opprimèrent les naturels du pays, allumèrent des guerres entre les différents États de cette vaste contrée, y répandirent la famine, la rébellion, la perfidie et tout ce déluge de maux qui afflige l'humanité. »

Dans la Métaphysique des moeurs, Kant récuse l'argument spécieux des conquérants qui invoquent la vacuité de territoires pour se les approprier, dans le cas de peuples chasseurs ou pasteurs, tout comme les justifications d'une conquête sous prétexte d'infériorité culturelle comme le firent les chrétiens en Allemagne, et continuaient de le faire les Russes en Sibérie ou les Européens en Amérique et en Afrique :

«Si cet établissement a lieu à une distance telle de la résidence du premier peuple qu'aucun des deux ne porte préjudice à l'autre dans l'usage de son territoire, le droit y relatif n'est pas douteux. Mais s'il s'agit de peuples pasteurs ou chasseurs (comme les Hottentots, les Toungouses et la plupart des nations américaines) qui pour subsister ont besoin de vastes contrées incultes, cette implantation ne saurait se faire par la violence mais seulement au moyen d'un contrat, qui lui-même ne profite pas de l'ignorance de ces indigènes quant à la cession de leurs terres, bien que soient apparemment suffisantes les justifications selon lesquelles une telle violence contribue à rendre le monde meilleur : en partie en amenant ces peuples grossiers à la culture (telle est l'allégation grâce à laquelle Büsching lui-même veut excuser l'introduction sanglante de la religion chrétienne en Allemagne), en partie en amenant son propre pays à se purifier d'hommes corrompus, et ceux-ci ou leurs descendants, à s'amender, comme on le leur souhaite, sur un autre continent (par exemple en Nouvelle Hollande); car toutes ces prétendues bonnes intentions n'arrivent pourtant pas à effacer l'injustice qui entache les moyens employés à les réaliser ${ }^{36}$. »

La menace que font peser les nations commerçantes de l'Europe sur le monde est explicite et Kant en a montré les conséquences destructrices pour les peuples et l'injustice. Ces expériences expliquent que la Chine et le Japon refusent ou contrôlent étroitement l'entrée des Européens sur leur territoire. La nature du commerce de l'Europe ne contribue pas au développement de relations amicales entre les peuples, mais bien au contraire invite ces derniers à l'éviter pour se protéger : 
« La Chine et le Japon, ayant appris à connaître par expérience les Européens, leur refusèrent sagement, sinon l'accès, du moins l'entrée de leur pays, à l'exception des Hollandais qu'ils excluent néanmoins, comme des captifs, de toute société avec les habitants. »

Kant espérait même que le moment de la disparition de ce commerce conquérant des Européens ne survivrait pas à la crise qu'il traversait alors et brosse un tableau très négatif de sa nature et de ses conséquences, non seulement dans le monde mais en Europe même. En effet, la crise de l'empire colonial en Amérique ouverte par l'indépendance des États-Unis avait entraîné des pertes importantes pour le commerce et les Compagnies des Indes. L'esclavage dans les plantations américaines est considéré par Kant comme une des formes les plus cruelles qui aient existé. Kant se démarque très clairement des tentatives de justification de la traite et de l'esclavage en Amérique par des colons qui le présentaient comme un mal nécessaire et une période de transition permettant aux Africains de se "civiliser" par cette nouvelle forme d'asservissement ${ }^{37}$ ! Est-il nécessaire de préciser que le rapprochement entre esclavage et civilisation est étranger à l'esprit des Lumières?

Du point de vue de la paix, Kant souligne que le commerce colonial, dont la traite des Africains fait partie, contribue au développement des flottes et des marins et nourrit donc l'arme par excellence de l'époque. Le commerce colonial accompagnant les conquêtes des puissances européennes sont des obstacles à la paix dans le monde. L'abolition de l'esclavage à Saint-Domingue en 1793, soutenue et élargie par la République française le 4 février 1794 et qui, au moment de la rédaction du Projet de paix perpétuelle, avait gagné la Guadeloupe et la Guyane et suscitait un renouveau des résistances des esclaves en Amérique, ouvrait une perspective de rupture avec les conquêtes des puissances européennes :

«Le pis, ou pour parler en moraliste, le mieux est que toutes ces violences sont en pure perte; que toutes les compagnies de commerce qui s'en rendent coupables touchent au moment de leur ruine; que les îles à sucre, ce repaire de l'esclavage le plus cruellement raffiné, ne produisent pas de revenu réel, et ne profitent qu'indirectement, ne servant même qu'à des vues peu louables, savoir à former des matelots pour les flottes, par conséquent à entretenir des guerres en Europe; service qu'en retirent surtout les puissances qui se targuent le plus de dévotion et qui, tout en s'abreuvant d'iniquités, prétendent égaler les élus en fait d'orthodoxie 38. »

Ces développements permettent de comprendre que le droit d'hospitalité soit conçu par Kant comme un simple droit de visite dans le but de protéger les peuples des dangers que représentent les conquêtes coloniales et plus particulièrement le commerce de domination des nations européennes.

Sur ce point, il est intéressant de comparer le projet de constitution présenté par SaintJust, le 24 avril 1793, à la Convention, en complément du projet de Déclaration des droits de l'homme et du citoyen de Robespierre ${ }^{39}$. Ce double projet de constitution développe une cosmopolitique de la liberté des peuples en vue de la paix en renonçant clairement à toute guerre offensive. Limitons-nous ici à préciser le thème du droit d'hospitalité défini par Saint-Just.

Le chapitre IX consacré aux relations extérieures définit un droit d'asile sélectif que la République française offre aux défenseurs de la liberté et aux opprimés de tous les pays, mais qu'elle refuse aux homicides et aux tyrans. Ce droit d'asile est par ailleurs offert à tous les navires étrangers dans les ports de la République qui s'engage aussi à 
ce que sa flotte porte secours aux navires en détresse et ouvre une diplomatie fondée sur les négociations avec les pays neutres et une réglementation de la guerre de course ${ }^{40}$ :

«Le peuple français se déclare l'ami de tous les peuples; il respectera religieusement les traités et les pavillons; il offre asile dans ses ports à tous les vaisseaux du monde; il offre un asile aux grands hommes, aux vertus malheureuses de tous les pays; ses vaisseaux protégeront en mer les vaisseaux étrangers contre les tempêtes... La République protège ceux qui sont bannis de leur patrie pour la cause de la liberté. Elle refuse asile aux homicides et aux tyrans ${ }^{41}$. "

À ce droit d'asile, Saint-Just ajoute un droit de visite des étrangers que la République s'engage à respecter, eux comme personnes et leurs usages : "Les étrangers et leurs usages seront respectés dans son sein.»

41 Saint-Just propose un droit d'accueil ou de séjour qui est un contrat de caractère réciproque établissant les mêmes droits aux Français établis à l'étranger et aux étrangers établis en France. Ce contrat crée un statut d'étranger établi dont l'exercice du droit de propriété est limité par l'autorisation de posséder des biens soit par héritage, soit par achat, mais avec l'interdiction de vendre : «Le Français établi en pays étrangers, l'étranger établi en France, peuvent hériter et acquérir; mais ils ne peuvent aliéner. »

42 Ce qui est visé est le commerce de domination : il s'agit d'empêcher les pratiques des négociants qui établissaient des membres de leur famille dans tous les lieux nécessaires à leur négoce qui structurait les politiques de conquête européennes. La République française annonce ainsi sa volonté de contrôler le négoce et, en l'interdisant aux Français établis à l'étranger, jette les bases d'accords réciproques avec d'autres États.

En proposant un droit d'asile aux amis de la liberté et aux vertus malheureuses, un droit de visite aux étrangers et une limitation de l'exercice du droit de propriété dans le but d'établir des rapports commerciaux contrôlés en vue de la paix, Saint-Just contribuait à définir un droit d'hospitalité sans le confondre avec une hostilité dangereuse sous couvert de pratiques commerciales dont on connaissait bien, à l'époque, les effets destructeurs de la liberté des peuples.

Cette comparaison avec le projet de Saint-Just permet d'avancer dans la compréhension du droit cosmopolitique défini par Kant comme restreint aux conditions de l'hospitalité universelle, en vertu du droit de commune possession de la surface de la terre qui n'est pas confondu avec une appropriation du sol.

Les trois niveaux juridiques définis plus haut - droit civil, droit des gens, droit cosmopolitique - sont essentiellement nécessaires pour que la paix perpétuelle puisse se matérialiser.

Le premier acte de la construction de la paix perpétuelle passe par la constitution d'un noyau d'États républicains ayant renoncé à la guerre de conquête et à la politique de puissance. L'allusion à la France est nette :

«La possibilité de réaliser cette idée d'une fédération, qui doit s'étendre insensiblement à tous les États et les conduire ainsi à la paix perpétuelle peut être démontrée. Car, si le bonheur voulait qu'un peuple puissant et éclairé se constituât en République (gouvernement qui, par sa nature, incline à la paix perpétuelle), il y aurait dès lors un centre pour cette alliance fédérative; d'autres États pourraient s'y joindre, afin d'assurer leur liberté, conformément à l'idée du droit des gens ${ }^{42}$. " 
Enfin, la publicité est la condition indispensable de la justice entre les hommes, car «toutes les actions relatives au droit d'autrui, dont la maxime n'est pas susceptible de publicité, sont injustes ${ }^{43}$ ". À tous ceux qui opposent la morale et la politique, et qui voudraient limiter leurs devoirs d'humanité à une "simple bienveillance" sans contenu juridique, Kant répond que la « simple bienveillance » ne peut fonder le droit.

Ainsi, Kant reprend et dépasse le débat philosophique des Lumières sur les relations entre les peuples. Le nouveau concept de droit cosmopolitique complète le droit civil et le droit des gens. Il fournit une réponse théorique achevée à l'interrogation iréniste ${ }^{44} \mathrm{de}$ l'époque moderne.

\section{NOTES}

1.Une partie de cet article reprend dans une forme modifiée des développements de la thèse de doctorat de M. BELISSA, soutenue à Paris I et publiée en version abrégée aux éditions Kimé, Fraternité universelle et Intérêt national (1713-1795). Les cosmopolitiques du droit des gens, Paris, 1998.

2.E. KANT, « Idées d'une Histoire universelle du point de vue cosmopolitique » VIII, 20-21 (1784) publié par PiobetTA S. La philosophie de l'Histoire de Kant, Paris, Denoël, 1947, p. 31. 3.Ibid., VIII 22, p. 33.

4.Ibid.

5.Ibid., p. 37, VIII 25.

6.La conception kantienne de l'état de guerre est donc très proche de celle de Locke. voir S.GOYARD-FABRE, «Qu'est-ce que l'état de guerre ? ", Cahiers de philosophie politique et juridique de l'Université de Caen, $\mathrm{n}^{\circ} 10,1986, \mathrm{pp} .147-161$.

7.S.GOYARD-FABRE, « Réflexions sur le pouvoir fédératif dans le « constitutionnalisme » de John Locke », Cahiers de philosophie politique et juridique de l'Université de Caen, $\mathrm{n}^{\circ} 5$, 1984, pp. 125-145.

8.Jean-Jacques ROUSSEAU, Fragment sur l'état de guerre dans Euvres, Paris, Bibliothèque de la Pléiade, 1964, tome III, p.610.

9.« Il faut reconnaître que le mot international est nouveau, bien qu'on puisse espérer qu'il soit suffisamment intelligible et analogique. Il est conçu pour exprimer, d'une manière plus significative, la branche du droit couramment appelée le droit des gens, une appellation si peu parlante, que, si ce n'était la force de la coutume, il semblerait plutôt s'appliquer à la jurisprudence interne " (notre traduction) An introduction to the principles of morals and legislation (1789) cité par P.GUGGENHEIM, Contribution à l'Histoire des sources du droit des gens, Leyde, 1959, p. 5. L'utilisation courante du néologisme de Bentham date du XIX ${ }^{\mathrm{e}}$ siècle.

10.KANT, Sur l'adage : cela est peut-être juste en théorie mais ne vaut pas pour la pratique dans Euvres philosophiques, Paris, Pléiade, 1986, tome III.

11.Ibid, VIII 307, p. 292.

12.ROBESPIERRE, Euvres choisies, (discours du 24 avril), Paris, Éditions sociales, 1973, tome II, pp. 135-136.Voir à ce sujet l'analyse de F. GAUTHIER, Triomphe et mort du droit naturel en 
Révolution, Paris, PUF, 1992, pp. 138-141 et M. BELISSA, Fraternité universelle (...), op. cit., p. 365 et « Robespierre et la guerre de conquête » dans De la Nation artésienne à la République et aux Nations, Actes du colloque Robespierre d'Arras, CHRN, Lille, 1993. 13.KANT, Sur l'adage : cela est peut-être juste en théorie mais ne vaut pas pour la pratique, dans op. cit., VIII 311-312, p. 297.

14.Le pouvoir fédératif est un concept lockéen. Dans la théorie constitutionnelle de Locke, il s'agit du pouvoir investi des relations entre les sociétés civiles. À ce titre, il est celui qui doit proclamer le droit entre les peuples. Voir M. BELISSA, « Droit des gens et théorie constitutionnelle dans la pensée des Lumières » dans Revue Historique de droit français et étranger, avril- juin 1998, pp. 215-233.

15.KANT, Sur l'adage : cela est peut-être juste en théorie mais ne vaut pas pour la pratique, dans op. cit., VIII 311, p. 298.

16. Voir M. BELISSA, « Droit des gens et théorie constitutionnelle dans la pensée des Lumières ", op. cit.

17.KANT, Sur l'adage : cela est peut-être juste en théorie mais ne vaut pas pour la pratique, dans op. cit., VIII 311-312, p. 297.

18.F. GAUTHIER, op. cit., p. 153.

19.Voir notamment DELBOS, «Les idées de Kant sur la paix perpétuelle », La Nouvelle Revue, 1899. FRIEDRICH C.J., « L' Essai sur la paix perpétuelle, sa position centrale dans la philosophie morale de Kant », Annales de philosophie politique, 1962, pp. 133-161. Le livre de Domenico losURDo, Autocensure et compromis dans la pensée politique de Kant, Presses Universitaires de Lille, 1993, est une démonstration sans faille de l'enracinement de la pensée politique du philosophe dans les événements révolutionnaires.

20.D. LOSURDO, op. cit., p. 175.

21.Joël LEFEBVRE, La Révolution française vue par les Allemands, Lyon, PUL, 1987, p. 10, cite les ouvrages sur la paix perpétuelle de Gentz, Krug, Herren, Friesen...

22.KANT, Projet de paix perpétuelle, Paris, Hatier, 1988, VIII 344-346, pp. 25-28.

23.Voir D.LOSURDO, op. cit., pp. 166-178.

24.KANT, Projet de paix perpétuelle, op. cit., VIII 344. La métaphore de l'arbre qui a ses propres racines pour évoquer la souveraineté d'un peuple et l'inaliénabilité de sa liberté se retrouve fréquemment à l'époque. Ainsi Louverture, lorsqu'il fut arrêté sur ordre du général Leclerc et déporté en France, dit à son vainqueur, dans son dernier message au peuple de Saint-Domingue : «En me renversant, on n'a abattu à SaintDomingue que le tronc de l'arbre de la liberté des Noirs ; il poussera par les racines, parce qu'elles sont profondes et nombreuses ", in Schoelcher, Vie de Toussaint Louverture, Paris, 1889, rééd. 1982, p. 349. Le contrat primitif du nouveau peuple de Saint-Domingue fondait effectivement le droit de liberté générale. Déjà, le 4 février 1794 lors de l'abolition de l'esclavage par la Convention, Danton l'avait exprimé dans ces mêmes termes : «En jetant la liberté dans le nouveau monde, elle y portera des fruits abondants, elle y poussera des racines profondes ", Archives parlementaires, CNRS, 1962, t. 84, p. 284 (16 pluviôse anII - 4 février 1794).

25.KANT, Métaphysique des mœurs, 1797, Doctrine du droit, VI 347.

26.Ibid., VI 348.

27.D. LOSURDO, op. cit., p. 127.

28.KANT, Métaphysique des mœurs, VI 330 pour les trois citations. Kant utilise le mot latin servus qui signifie esclave, bien que la traduction de la Pléiade donne " serf ».

29.KANT, Projet de paix perpétuelle, op. cit., VIII 349, p. 37. 
30.Ibid., VIII 349, p. 31.

31.Kant revient sur cette question dans la Métaphysique des moeurs de 1797. L'idée qu'il existe une « communauté pacifique sinon encore amicale de tous les peuples de la terre » n'est pas « philanthropique » (éthique) mais c'est un principe juridique. Il s'agit d'une perspective plutôt que d'une réalité : « On peut dire que cette institution universelle et perpétuelle de la paix, n'est pas une simple partie, mais constitue la fin ultime tout entière de la doctrine du droit dans les limites de la simple raison ", CEuvres philosophiques, op. cit., VI 354-355, p. 629.

32.G. F. DE MARTENS, Précis du droit des gens moderne de l'Europe, Paris, 1864 (traduction de 1'édition de 1796). Dans le même ouvrage, Martens critique violemment la déclaration du droit des gens de l'abbé Grégoire dont les principes sont proches de ceux développés par Kant.

33.KANT, Projet de paix perpétuelle, op. cit., VIII 360, p. 43.

34.Ibid., VIII 358.

35.Ibid.

36.Id., Métaphysique des mœurs, Le droit cosmopolitique, VI, 353. Büsching (1724-1793) était un géographe et un historien.

37.Voir par exemple l'intervention de Cocherel, député des colons de Saint-Domingue à la Constituante, le 26 novembre 1789, en faveur du maintien de l'esclavage dans les colonies et qui justifiait la traite comme un moyen de soustraire « des Africains au plus dur esclavage, qui fait la base et la constitution indestructible de ce (sic) peuple barbare ", Archives parlementaires, t. 10, p. 263.

38. KANT, op. cit., VIII 359. Sur la critique du colonialisme par Kant, voir D. LOSURDO, op. cit., pp. $171 \mathrm{sq}$.

39. ROBESPIERRE, Déclaration des droits de l'homme et du citoyen, Imp. nat., Chambre des députés, Collection Portiez de l'Oise, t. XXIX, n 42, reproduit dans ROBESPIERRE, Euvres, t. IX, PUF, 1958, pp. 463 sq. Le texte de Saint-Just est reproduit dans Théorie politique, éd. de A. Liénart, Paris, Seuil, 1976, pp. 197 sq.

40.Voir à ce sujet M. BELISSA, Fraternité universelle (...), op. cit., IIIe partie, chap. 3, p. 369.

41.SAINT-JUST, op. cit., articles un, quatre et cinq.

42.KANT, Projet de paix perpétuelle, op. cit., p. 39.

43.Ibid., p. 68.

44.De eirênê, la paix en grec, le terme de « pacifiste » est selon nous anachronique.

\section{RÉSUMÉS}

La proposition kantienne d'un droit cosmopolitique cherche à résoudre le problème des conditions de réalisation de la liberté, dans une perspective de paix, entre les personnes et entre les peuples. Si la liberté est un droit propre à l'humanité, c'est-à-dire réciproque ou universel, la possibilité de sa réalisation suppose que soient pensés conjointement, et non séparément, le droit civique, le droit des gens et le droit cosmopolitique. Une telle perspective de paix n'est pas seulement un état de non-guerre, mais un état juridique à construire, emportant le devoir de renoncer aux politiques de puissance conquérante ou colonialiste et à ce qui les fonde : armées 
permanentes, pouvoir exécutif hégémonique, économie de domination des nations commerçantes de l'Europe en particulier. Se dégage alors un droit d'hospitalité conçu par Kant comme un droit de visite qui appartient à tout être humain, mais qui ne saurait être confondu avec les pratiques dangereuses d'une appropriation du sol, des richesses ou des habitants.

Kant, Cosmopolitical Law and the Civil Society of Nations. Kant's proposed cosmopolitical law attempts to resolve the problem of how to achieve liberty with a view to securing peace between persons and peoples. While liberty is a right peculiar to mankind, i. e. reciprocal or universal, the possibility of achieving it presupposes a fusion, not a separation, of civil law, the law of nations and cosmopolitan law. Such a prospect of peace is not just a state of non-war, but a juridical state which needs to be built, entailing the obligation to repudiate conquest, colonialism and power politics, together with their underpinnings : standing armies, hegemonic executives, economies of domination, on the part of European trading nations in particular. From this there emerges a right to hospitality which Kant conceives of as a visiting right belonging to every human being, but not to be confused with dangerous practices such as the appropriation of land, riches or native inhabitants.

La proposta kantiana di un diritto cosmopolita cerca di risolvere il problema delle condizioni di attuazione della libertà, in una prospettiva di pace, tra le persone e tra i popoli. Se la libertà è un diritto propriamente umano, vale a dire reciproco o universale, la possibilità della sua attuazione implica che siano pensati contemporaneamente e non separatamente, il diritto civile, il diritto delle persone e il diritto cosmopolitico. Tal prospettiva di pace non è solo uno stato di nonguerra, bensì uno stato giuridico da costruire che necessita il dovere di rinunciare alle politiche di potenza conquistatrice o colonialista nonchè quello su cui esse si fondano: eserciti permanenti, potere esecutivo egemonico, economia di dominazione delle nazioni commercianti dell'Europa in particolare. Emmerge allora un diritto di ospitalità concepito da Kant come un diritto di visita che appartiene ad ogni essere umano, ma che non va confuso con le pratiche pericolose di un'appropriazione del suolo, delle ricchezze o degli abitanti.

La propuesta kantiana de un derecho cosmopolítico intenta resolver el problema de las condiciones de realización de la libertad entre las personas y los pueblos, en una perspectiva de paz. Si la libertad es un derecho propio de la humanidad, es decir recíproco o universal, la posibilidad de su realización supone una reflexión sobre el derecho cívico, el derecho de la gante y el derecho cosmopolítico. Esa perspectiva de paz no es solamente un estado de no-guerra, pero un estado jurídico a construir, un estado que tiene el deber de renuciar a las políticas de potencia conquistadora o colonialista y a lo que la funda: ejércitos permanentes, poder ejecutivo hegemonístico, economía de dominación de las naciones comerciantes de Europa en particular. Aparece así un derecho de hospitalidad concebido por Kant como un derecho de visita perteneciente a todo ser humano, pero que no puede concebirse con las prácticas peligrosas de una apropiación del suelo, de las riquezas o de los habitantes.

Der kantische Vorschlag eines Weltbürgerrechts ist ein Versuch, das Problem der Entstehungsbedingungen der Freiheit in der Perspektive eines Friedens zwischen den Menschen und den Völkern zu lösen. Wenn die Freiheit ein wesentliches Recht der Menschheit ist, setzt die Möglichkeit ihrer Verwirklichung voraus, daß das Staats - das Völker - und das Weltbürgerrecht zusammen und nicht getrennt gedacht werden sollen. Eine solche Friedensperspektive ist nicht nur die Abwesenheit von Krieg, sondern ein zu konstuierender Zustand, der die Pflicht enthält, auf eine Eroberungs - oder Kolonialpolitik und auf alles, was diese stützt, zu verzichten, d. h. auf stehende Heere, auf eine auf Hegemonie ausgerichtete Exekutive oder auf die herrschsüchtige Wirtschaft insbesondere der europäischen Handelsnationen. Daraus entsteht nun ein Gastrecht, das Kant als ein Besuchsrecht versteht, das jedem Menschen eigen ist, das jedoch keineswegs mit 
der gefährlichen Praxis einer Aneignung des Bodens, der Reichtümer oder der Einwohner zu verwechseln wäre.

\section{AUTEURS}

MARC BELISSA

Université de Nantes

FLORENCE GAUTHIER

Université de Paris VII 